\title{
Dependence of kink oscillation damping on the amplitude
}

\author{
C. R. Goddard ${ }^{1}$ and V. M. Nakariakov ${ }^{1,2,3}$ \\ ${ }^{1}$ Centre for Fusion, Space and Astrophysics, Department of Physics, University of Warwick, CV4 7AL, UK \\ e-mail: c.r.goddard@warwick.ac.uk \\ 2 Astronomical Observatory at Pulkovo of the Russian Academy of Sciences, 196140 St. Petersburg, Russia \\ 3 School of Space Research, Kyung Hee University, 446-701 Yongin, Gyeonggi, Korea
}

Received 14 April 2016/ Accepted 4 May 2016

\begin{abstract}
Context. Kink oscillations of coronal loops are one of the most intensively studied oscillatory phenomena in the solar corona. In the large-amplitude rapidly damped regime, these oscillations are observed to have a low quality factor with only a few cycles of oscillation detected before they are damped. The specific mechanism responsible for rapid damping is commonly accepted to be associated with the linear coupling between collective kink oscillations and localised torsional oscillations, the phenomenon of resonant absorption of the kink mode. The role of finite amplitude effects, however, is still not clear.

Aims. We investigated the empirical dependence of the kink oscillation damping time and its quality factor, which is defined as the ratio of damping time to oscillation period, on the oscillation amplitude.

Methods. We analysed decaying kink oscillation events detected previously with TRACE, SDO/AIA and and STEREO/EUVI in the extreme ultraviolet (EUV) $171 \AA$ A band.

Results. We found that the ratio of the kink oscillation damping time to the oscillation period systematically decreases with the oscillation amplitude. We approximated the quality factor dependence on the oscillation displacement amplitude via the power-law dependence with the exponent of $-1 / 2$, however we stress that this is a by-eye estimate, and a more rigorous estimation of the scaling law requires more accurate measurements and increased statistics. We conclude that damping of kink oscillations of coronal loops depends on the oscillation amplitude, indicating the possible role of non-linear mechanisms for damping.
\end{abstract}

Key words. Sun: oscillations - Sun: corona - methods: observational

\section{Introduction}

Kink oscillations of coronal loops, which are transverse displacements of the loops typically seen in the extreme ultraviolet (EUV) band, are one of the most intensively studied oscillatory phenomena in the solar corona (e.g. Liu \& Ofman 2014; Nakariakov et al. 2016, for recent comprehensive reviews). Typical oscillation periods are of several minutes (e.g. Zimovets \& Nakariakov 2015; Goddard et al. 2016, for recent statistics). Kink oscillations are observed in two regimes, quickly damped, typically in a few cycles, and undamped (e.g. Nisticò et al. 2013). The damped oscillations are typically seen to have larger amplitudes, of several Mm, comparable to or exceeding the minor radii of the oscillating loops. Recently, it was shown that in the majority of cases kink oscillations are excited by low coronal eruptions that mechanically displace the loops from the equilibrium (Zimovets \& Nakariakov 2015).

The rapid decay of kink oscillations has been explained in terms of linear coupling of the collective kink (fast magnetoacoustic) mode to torsional (shear) Alfvén waves in a narrow resonant layer, where the phase speed of the kink wave matches the local Alfvén speed (e.g. Ruderman \& Roberts 2002; Goossens et al. 2002), which is the effect of resonant absorption of kink non-axisymmetric magnetohydrodynamic (MHD) oscillations in a transversely non-uniform plasma. In this mechanism, the energy of kink oscillations transfers to the unresolved torsional Alfvénic motions (e.g. Goossens et al. 2011). This mechanism has been shown to produce damping profiles and rates consistent with observational results (e.g. Verth et al. 2010; Pascoe et al. 2016).

The majority of theoretical studies of kink oscillations were performed in the linear regime and, in these studies, the finite amplitude effects were neglected in the governing MHD equations. Indeed, in kink oscillations the amplitudes of the perturbations of the magnetic field and density, as well as the speeds of the displacement, are observed to be just a few percent of the equilibrium parameters and the Alfvén speed, respectively, justifying the linear nature of the oscillation. On the other hand, the displacement amplitude is comparable to the loop minor radius, suggesting that the assumption of the linearity of the observed kink oscillations might not be fully applicable to all oscillating loops. Moreover, the resultant shear Alfvén waves may become large amplitude because of their very narrow localisation at the resonant shell inside the oscillating loop and their inability to spread across the field. Recently, however, consideration of the time-dependent evolution of this process has shown that the shear Alfvén waves are not exclusively confined to the hypothetical narrow resonant layer, but spread along the whole nonuniform layer because of phase mixing, gradually lowering their resulting amplitude (e.g. Soler \& Terradas 2015). Non-linearity may modify the efficiency of well-known damping mechanisms or introduce additional sources of damping or dissipation. But, with the lack of direct observational evidence of non-linear effects in the kink mode dynamics, theory of non-linear kink oscillations has only been addressed in several dedicated studies.

In loops with the step-function transverse profile, i.e. in which the boundaries are tangential discontinuities, the effect of 
resonant absorption does not occur unless the magnetic field is twisted. In the straight magnetic field case, analysis of weakly non-linear effects revealed the possibility of resonant non-linear coupling of kink modes with modes of different azimuthal symmetry, such as the sausage mode (e.g. Nakariakov \& Oraevsky 1995), and the non-linear modification of the resonant frequency that becomes dependent on the oscillation amplitude squared (Ruderman \& Goossens 2014).

Several theoretical studies have generalised the effect of resonant absorption in the non-linear regime. For example, large amplitude kink waves can induce field-aligned plasma flows and density perturbations by the ponderomotive force, which is similar to the well-known non-linear effect in linearly or elliptically polarised Alfvén waves (see Vasheghani Farahani et al. 2012, for a recent discussion). For example, Terradas \& Ofman (2004) showed that this effect leads to the accumulation of mass at the loop top. The resulting redistribution of the matter in the oscillating loop would change the location of resonant layers and, hence, the efficiency of wave damping. These induced flows are usually essentially sub-sonic and sub-Alfvénic, as they are proportional to the square of the relative amplitude of the mother kink waves. However, these induced flows are likely to be nonuniform in the transverse direction (e.g. Clack \& Ballai 2009). These flows may cause various shear-flow instabilities that enhance the transport coefficients locally and, hence, the damping (e.g. Ofman \& Davila 1995).

Ofman et al. (1994) showed that the Kelvin-Helmholtz instability (KHI) for torsional Alfvén waves, first described by Browning \& Priest (1984) in the context of Alfvén phase mixing, can occur at the resonance layer of the oscillating loop, thereby resulting in enhanced dissipation. Terradas et al. (2008) performed a high-resolution, three-dimensional numerical study of non-linear kink oscillations. Ofman et al. found that shearflow instabilities develop and deform the boundary of the flux tube, and the evolution of the tube is very sensitive to the amplitude of the initial perturbation. They relate their results to the development of KHI. It was found that KHI can develop in timescales comparable to the kink oscillation period (Soler et al. 2010).

Ruderman et al. (2010) considered weakly non-linear kink waves in a tube filled in with an incompressible plasma, and concluded that non-linearity accelerates the wave damping as a result fo resonant absorption. On the other hand, works of Clack \& Ballai (2009) and Ballai \& Ruderman (2011) demonstrated that the linear approach can give rather accurate results with no need for using a mathematically more cumbersome nonlinear description. More recently Ruderman \& Goossens (2014) studied non-linear kink oscillations of coronal loops. The nonlinear correction to the oscillation frequency was calculated, which is proportional to the square of the amplitude, and it was again found that non-linearity can strongly enhance resonant damping.

Thus, despite significant progress in the development of the non-linear theory of kink oscillations, there is still no a clear picture of the quantitative effect of the finite amplitude on the behaviour and damping of these oscillations. Further progress in understanding the role of the non-linearity and the exploitation of its seismological potential requires observational guidance. In this work, we aim to establish empirically the relationship between the damping time and amplitude of kink oscillations. In Sect. 2 we describe the data used and the method of the analysis. In Sect. 3 we describe the results. Our findings are summarised and discussed in Sect. 4.
Table 1. Damping times, periods, and apparent amplitudes of kink oscillations of coronal loops.

\begin{tabular}{|c|c|c|c|}
\hline $\begin{array}{l}\text { Ref. } \\
(\#)\end{array}$ & $\begin{array}{l}\text { Damping time } \\
\text { (s) }\end{array}$ & $\begin{array}{l}\text { Period } \\
\text { (s) }\end{array}$ & $\begin{array}{l}\text { Amplitude } \\
(\mathrm{Mm})\end{array}$ \\
\hline 1 & 870 & 261 & 0.8 \\
\hline 1 & 300 & 265 & 2 \\
\hline 1 & 500 & 316 & 6 \\
\hline 1 & 400 & 277 & 4 \\
\hline 1 & 849 & 272 & 5 \\
\hline 1 & 600 & 435 & 0.7 \\
\hline 1 & 200 & 143 & 0.5 \\
\hline 1 & 800 & 423 & 0.7 \\
\hline 1 & 200 & 185 & 9 \\
\hline 1 & 400 & 396 & 1.8 \\
\hline 2 & 714 & 234 & 7.9 \\
\hline 3 & 920 & 249 & 0.34 \\
\hline 3 & 1260 & 448 & 0.43 \\
\hline 3 & 1830 & 392 & 0.49 \\
\hline 3 & 1330 & 382 & 0.42 \\
\hline 3 & 1030 & 358 & 0.56 \\
\hline 3 & 980 & 326 & 0.22 \\
\hline 3 & 1320 & 357 & 0.24 \\
\hline 4 & 2129 & 436 & 0.4 \\
\hline 4 & 1200 & 243 & 0.4 \\
\hline 5 & 521 & 895 & 7.3 \\
\hline 5 & 473 & 452 & 3.7 \\
\hline 6 & 1000 & 630 & 3.7 \\
\hline 7 & 3660 & 2418 & 5 \\
\hline 8 & 500 & 377 & 9.5 \\
\hline
\end{tabular}

Notes. The data listed were previously detected using TRACE and STEREO/EUVI. The first column indicates the publication the results are taken from:

References. 1: Aschwanden et al. (2002); 2: Wang \& Solanki (2004); 3: Verwichte et al. (2004); 4: Van Doorsselaere et al. (2007); 5: De Moortel \& Brady (2007); 6: Verwichte et al. (2009); 7: Verwichte et al. (2010); 8: Mrozek (2011).

\section{Observations and analysis}

Our analysis is based on the observational results summarised in the catalogue created by Zimovets \& Nakariakov (2015). The decaying kink oscillations included in that catalogue were obtained using the $171 \AA$ channel of SDO/AIA. Parameters of the kink oscillations used in the present study are shown in Table A.1 of Goddard et al. (2016). This data was supplemented by the other previously published events detected with TRACE and STEREO/EUVI. The damping time, period, and apparent (projected) amplitude of the oscillations were retrieved where possible. Some published detections had to be omitted as the amplitude of the oscillation was not reported and could not be reliably estimated from the provided figures. These additional data are summarised in Table 1.

In all of the cases, the estimations of the oscillation parameters were made with the use of time-distance plots made for slits positioned across the local axis of the oscillating loop at the locations of the highest apparent amplitude. The initial displacement was defined as the difference between the initial loop position and the first maximum, and the initial amplitude was defined between the first maximum and minimum. The reported displacements and amplitudes are not absolute measurements due to the unknown angle between the line of sight (LoS) and the direction of the oscillatory displacement. 


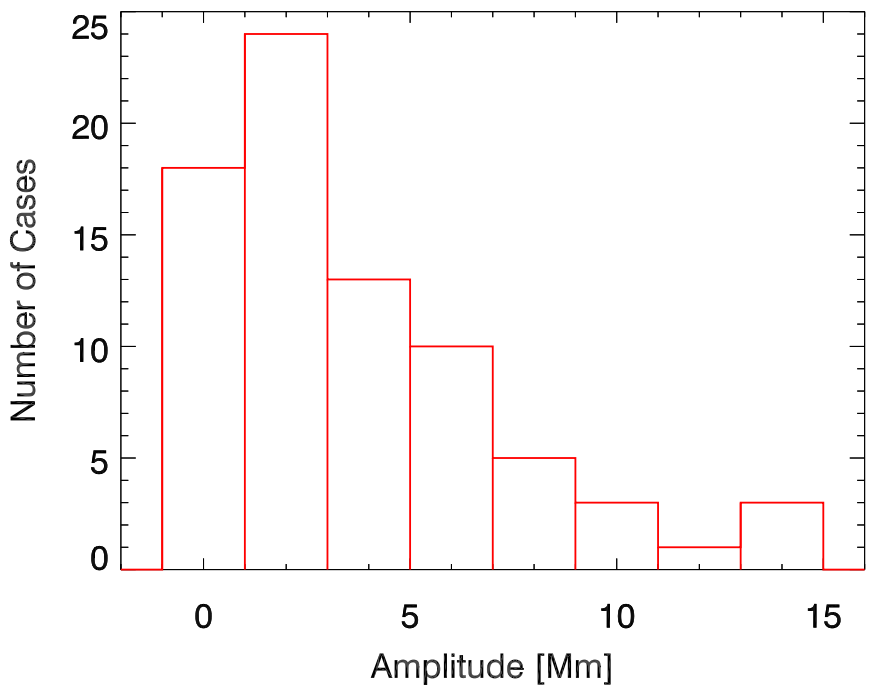

Fig. 1. Distribution of the measured apparent initial amplitude of kink oscillations of coronal loops, detected with SDO/AIA, TRACE, and STEREO/EUVI. The bin size is $2 \mathrm{Mm}$.

The kink oscillation periods reported in Goddard et al. (2016) were obtained by best fitting a sinusoid to the detrended oscillation and taking the best fitting period and its associated error. For oscillations that showed clear damping, the exponential damping time and error were obtained by fitting the maxima of the absolute value of the detrended signal. Parts of the signal that appeared to have non-exponential damping envelopes were excluded from the fitting.

\section{Results}

Figure 1 shows the distribution of the observed apparent amplitudes of kink oscillations. This figure is a modification of Fig. 5 of Goddard et al. (2016), adding the results obtained for the events shown in Table 1. There appears to be no significant difference between these two figures, other than fewer statistics, as only about half of the events discussed in Goddard et al. (2016) are used in this work. Also, in the current figure, the highest amplitude tail is absent, as in that part of the distribution the oscillations do not have enough cycles to measure damping.

In Fig. 2 the quality factor of the kink oscillations, defined as the ratio of the damping time to the period, is plotted against the apparent amplitude of the oscillatory displacement. A negative dependence between the two parameters is evident, with the Spearman and Pearson correlation coefficients of -0.44 and -0.42 , respectively. These have p-values of $6 \times 10^{-5}$ and $5 \times 10^{-5}$, respectively. It is clear that larger amplitudes correspond to systematically smaller quality factors of the oscillations. The dependence has a triangular shape on the quality factor - apparent amplitude plane. For lower apparent amplitudes, up to 3$4 \mathrm{Mm}$, the quality factor ranges from 1 to 5 , while for higher amplitudes the range of the measured quality factors decreases to from 1 to 2. Unfortunately, different methods of data analysis applied in the different studies summarised in Table 1 make it difficult to estimate the error bars of those measurements (blue points in Fig. 2).

The upper boundary of the data cloud in Fig. 2 can be approximated by the expression

$q \approx 7 \times A[\mathrm{Mm}]^{-1 / 2}$,

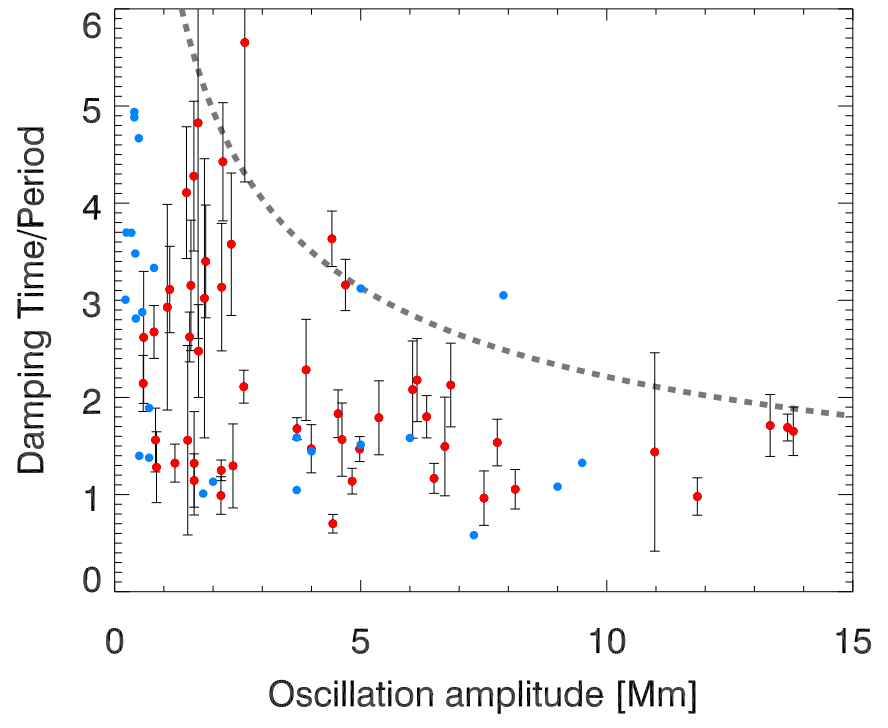

Fig. 2. Quality factor of kink oscillations of coronal loops determined as the ratio of damping time to the oscillation period, plotted against the apparent oscillation amplitude. The red points are taken from Goddard et al. (2016). The blue points correspond to those listed in Table 1 . The grey line shows the scaling of the quality factor with the maximum apparent (actual) amplitude.

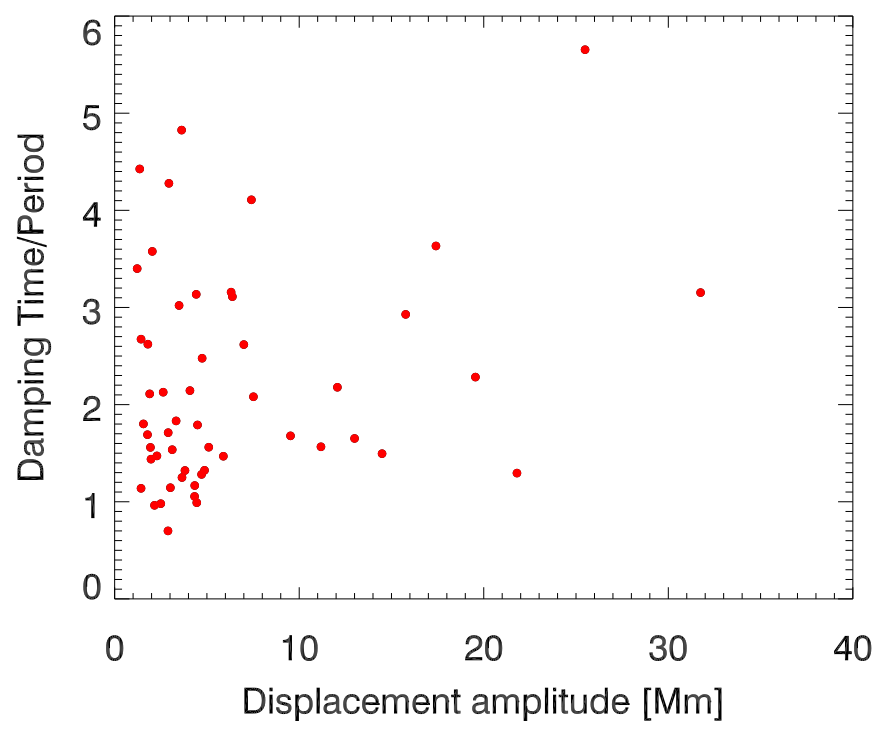

Fig. 3. Quality factor of kink oscillations of coronal loops (the ratio of damping time to period) plotted against the apparent amplitude of the initial displacement that excited the oscillation. The data is taken from Goddard et al. (2016).

where $q$ is the quality factor and $A$ is the kink oscillation amplitude. In the lack of a large number of observations this bestfitting curve was made by eye, and should only be considered a first attempt to quantify this scaling. A fit to the main body of the data was not considered to be significant as this is affected by the suppression of the amplitudes by the unknown LoS angle, which is discussed further in Sect. 4.

In Fig. 3 the quality factor of the oscillations is plotted against the apparent amplitude of the initial displacement that excited the oscillation. In contrast to Fig. 2, we see no correlation despite the weak correlation between the amplitude and displacement reported in Goddard et al. (2016). This indicates that the amplitude of the oscillation itself, rather than amplitude of 
the initial displacement, affects the quality factor and, therefore, the damping of the oscillations.

\section{Discussion and conclusions}

The triangular shape of the data cloud on the quality factor - apparent amplitude plane has a simple interpretation. The apparent amplitude is proportional to the actual amplitude reduced by the angle between the LoS and the direction of the oscillatory displacement. Thus, if one observes several kink oscillations of the same displacement amplitude, but randomly distributed with respect to the LoS angle, the apparent amplitudes would range from zero (or the detection threshold determined e.g. by the pixel size), for those oscillations that oscillated along the LoS, to the actual amplitude, for the oscillations displacing the loop in the plane of sky. In other words, for a given value of the quality factor, the apparent amplitudes of kink oscillations measured with randomly distributed LoS angles are distributed in a horizontal stripe in the quality factor - apparent amplitude plane, from zero to the actual amplitude. Figure 2 shows that for higher quality factors the highest apparent amplitudes are systematically lower. Thus, the triangular shape of the data cloud clearly demonstrates the decrease in the quality factor with the amplitude. We approximated the quality factor dependence on the oscillation displacement amplitude by the power-law dependence with the exponent of $-1 / 2$, however we stress that this is a by eye estimate, and a more rigorous estimation of the scaling law requires more accurate measurements and increased statistics. The physical mechanism responsible for this dependence, and consistent with this constraint, needs to be revealed.

The same reasoning is applicable to the dependence of the quality factor on the apparent initial displacement amplitude. This dependence is more scattered than the quality factor, making it difficult to establish a relationship between the quality factor and the initial displacement. Thus, we conclude that our data do not allow us to determine any scaling of these two parameters of kink oscillations. We should stress that the initial displacement and oscillation amplitude of kink oscillations are different parameters, as the loop can oscillate around a new equilibrium after the initial displacement (Zimovets \& Nakariakov 2015). The difference between the initial displacement and the observed amplitude could also be attributed to the excitation of leaky modes and higher harmonics, which was numerically found by Terradas et al. (2007) who considered the excitation of kink oscillations by a magnetic pressure pulse.

Thus, the main finding of this work is the demonstration of the dependence of the kink oscillation quality factor on the oscillation amplitude. This result indicates that the damping mechanism depends upon the amplitude and hence is non-linear. The dependence seems to be smooth, with a gradual decrease in the quality factor with the amplitude, and does not have a clear break that would indicate the presence of a threshold typical for shearflow instabilities, such as KHI. However, the lack of break may be attributed to insufficient statistics, and the use of a larger set of oscillatory events could change this conclusion.

Acknowledgements. The work was supported by the European Research Council under the SeismoSun Research Project No. 321141 (CRG, VMN), the STFC consolidated grant ST/L000733/1, and the BK21 plus programme of the National Research Foundation funded by the Ministry of Education of Korea.

\section{References}

Aschwanden, M. J., de Pontieu, B., Schrijver, C. J., \& Title, A. M. 2002, Sol. Phys., 206, 99

Ballai, I., \& Ruderman, M. S. 2011, Space Sci. Rev., 158, 421

Browning, P. K., \& Priest, E. R. 1984, A\&A, 131, 283

Clack, C. T. M., \& Ballai, I. 2009, Phys. Plasmas, 16, 072115

De Moortel, I., \& Brady, C. S. 2007, ApJ, 664, 1210

Goddard, C. R., Nisticò, G., Nakariakov, V. M., \& Zimovets, I. V. 2016, A\&A, 585, A 137

Goossens, M., Andries, J., \& Aschwanden, M. J. 2002, A\&A, 394, L39

Goossens, M., Erdélyi, R., \& Ruderman, M. S. 2011, Space Sci. Rev., 158, 289 Liu, W., \& Ofman, L. 2014, Sol. Phys., 289, 3233

Mrozek, T. 2011, Sol. Phys., 270, 191

Nakariakov, V. M., \& Oraevsky, V. N. 1995, Sol. Phys., 160, 289

Nakariakov, V. M., Pilipenko, V., Heilig, B., et al. 2016, Space Sci. Rev., 200, 75

Nisticò, G., Nakariakov, V. M., \& Verwichte, E. 2013, A\&A, 552, A57

Ofman, L., \& Davila, J. M. 1995, J. Geophys. Res., 100, 23427

Ofman, L., Davila, J. M., \& Steinolfson, R. S. 1994, Geophys. Res. Lett., 21, 2259

Pascoe, D. J., Goddard, C. R., Nisticò, G., Anfinogentov, S., \& Nakariakov, V. M. 2016, A\&A, 585, L6

Ruderman, M. S., \& Goossens, M. 2014, Sol. Phys., 289, 1999

Ruderman, M. S., \& Roberts, B. 2002, ApJ, 577, 475

Ruderman, M. S., Goossens, M., \& Andries, J. 2010, Phys. Plasmas, 17, 082108

Soler, R., \& Terradas, J. 2015, ApJ, 803, 43

Soler, R., Terradas, J., Oliver, R., Ballester, J. L., \& Goossens, M. 2010, ApJ, 712,875

Terradas, J., \& Ofman, L. 2004, ApJ, 610, 523

Terradas, J., Andries, J., \& Goossens, M. 2007, A\&A, 469, 1135

Terradas, J., Andries, J., Goossens, M., et al. 2008, ApJ, 687, L115

Van Doorsselaere, T., Nakariakov, V. M., \& Verwichte, E. 2007, A\&A, 473, 959

Vasheghani Farahani, S., Nakariakov, V. M., Verwichte, E., \& Van Doorsselaere, T. 2012, A\&A, 544, A127

Verth, G., Terradas, J., \& Goossens, M. 2010, ApJ, 718, L102

Verwichte, E., Nakariakov, V. M., Ofman, L., \& Deluca, E. E. 2004, Sol. Phys., 223, 77

Verwichte, E., Aschwanden, M. J., Van Doorsselaere, T., Foullon, C., \& Nakariakov, V. M. 2009, ApJ, 698, 397

Verwichte, E., Foullon, C., \& Van Doorsselaere, T. 2010, ApJ, 717, 458

Wang, T. J., \& Solanki, S. K. 2004, A\&A, 421, L33

Zimovets, I. V., \& Nakariakov, V. M. 2015, A\&A, 577, A4 